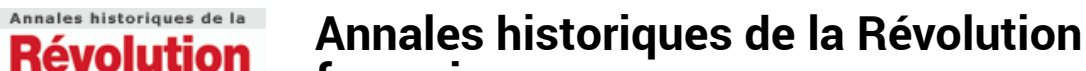 \\ française française
}

319 | janvier-mars 2000

Varia

\section{L'ordre politique chez Sieyès en l'an III}

\section{Andreï Tyrsenko}

\section{(2) OpenEdition}

\section{Journals}

\section{Édition électronique}

URL : https://journals.openedition.org/ahrf/100

DOI : 10.4000/ahrf.100

ISSN : $1952-403 X$

\section{Éditeur :}

Armand Colin, Société des études robespierristes

\section{Édition imprimée}

Date de publication : 1 mars 2000

Pagination : 27-45

ISSN : 0003-4436

\section{Référence électronique}

Andreï Tyrsenko, «L'ordre politique chez Sieyès en l'an III », Annales historiques de la Révolution

française [En ligne], 319 | janvier-mars 2000, mis en ligne le 11 mai 2006, consulté le 23 avril 2022

URL : http://journals.openedition.org/ahrf/100 ; DOI : https://doi.org/10.4000/ahrf.100

Ce document a été généré automatiquement le 23 avril 2022.

Tous droits réservés 


\section{L'ordre politique chez Sieyès en l'an III $^{1}$}

\section{Andreï Tyrsenko}

1 L'originalité de la pensée politique de Sieyès procède d'abord d'idées avancées de son propre chef au cours des débats immédiatement antérieurs et contemporains de l'année 1789. Puis, Sieyès a proposé une série de projets constitutionnels en appui sur sa conception générale de l'ordre social : il a donc précisé, dans ces projets, ses idées initiales. De ce point de vue, la pensée politique de Sieyès en l'an III présente un très grand intérêt si l'on veut dresser le bilan des idées d'un des auteurs majeurs de la Révolution française, et de leur implication immédiate dans l'expérience révolutionnaire.

2 C'est au sein même de la Convention que Sieyès a avancé ses propositions concernant la future Constitution, élaborée par la Commission des Onze, dans deux textes à forte valeur théorique, l'Opinion de Sieyès sur plusieurs articles des titres IV et $V$ du projet de Constitution et l'Opinion de Sieyès sur les attributions du jury constitutionnaire ${ }^{2}$. Mais nous disposons aussi, dans le fonds Sieyès des Archives Nationales ${ }^{3}$, de documents manuscrits précisant les vues politiques de Sieyès au même moment. Sans les négliger dans leur ensemble, nous mettons surtout l'accent sur le manuscrit qui nous semble être le plus important, intitulé : «Bases de l'ordre social ou série raisonnée de quelques idées fondamentales de l'état social et politique.An $3 »^{4}$ : nous y trouvons un exposé systématique de la doctrine de Sieyès sous-jacente à sa conception du futur ordre constitutionnel en France.

3 Nous nous proposons donc, à partir de telles sources imprimées et manuscrites, de donner une vue d'ensemble de la pensée politique de Sieyès en l'an III.

4 Réfléchissant sur l'ordre social que l'on doit instaurer en France, Sieyès fait immédiatement référence aux Droits de l'homme comme bases de tout ordre social :

«Une constitution n'est point une transaction entre les volontés arbitraires. Tout découle des Droits de l'homme et y aboutit par un enchaînement de vérités nécessaires. Hors de là, il ne peut y avoir que Théocratie, machiavélisme ou Brigandage. $»^{5}$ 

relever, dans ses fondements, d'une démarche déductive et analytique : "L'ordre social ne peut être établi que sur une suite de vérités ? Où prendre la première, celle qui doit servir de fondement ? ${ }^{6}$ De fait Sieyès ne récuse pas la méthode rationaliste, mais s'oppose à toute explication «métaphysique scolastique» qui privilégie le raisonnement sur la méthode analytique. "La société des hommes n'est point une affaire de raisonnement ", précise-t-il ${ }^{7}$. En proposant une métaphysique de l'activité du moi, constitutive d'un "ordre du moi ", Sieyès se démarque d'une approche essentialiste des Droits de l'homme. Il s'appuie ainsi sur la figure du philosophe, "spectateur philosophe» à la veille de la Révolution française, puis "philosophe analyste » en l'an III. La " métaphysique politique », qu'il revendique haut et fort dans son Préliminaire à la Constitution française (1789) procède de l'affirmation que la politique se fonde sur la philosophie. S'il n'est plus question de raisonnement, sans doute à l'encontre des Physiocrates, il s'agit plutôt de privilégier l'observation analytique dans l'exposition raisonnée des Droits de l'homme.

La complexité de la pensée de Sieyès tient au fait qu'il conserve la dimension ontologique du rationalisme (Descartes), revu à travers le dynamisme leibnizien, tout en tenant compte des apports de la pensée empiriste anglo-écossaise relative à la formation de la société civile sur la base de ses besoins et de ses moyens. Attitude contradictoire en apparence seulement, dans la mesure où l'on peut trouver dans la métaphysique cartésienne une connexion entre l'ontologie rationnelle de l'idée de Dieu et la dimension rationnelle des choses et des hommes ${ }^{8}$, donc une phénoménologie du vécu, une dimension subjective immanente de la rationalité que "l'école anglaise » saura faire fructifier. C'est ainsi que le traitement par Locke des idées de Descartes a permis l'enrichissement de la voie empirique vers la compréhension des phénomènes civils. Condillac s'inscrit dans cette lignée : il ne faut donc pas s'étonner que Sieyès en fasse l'une de ses lectures critiques majeures au sein du cahier métaphysique ${ }^{9}$, principal de ses manuscrits philosophiques. Ainsi Sieyès veut rendre compte à la fois du mouvement intellectuel du moi, d'une politique des besoins de l'homme, de l'art social comme ensemble d'effets et de moyens adéquats à la construction de l'ordre social pour en fin de compte concevoir une articulation harmonieuse entre la liberté individuelle et l'ordre représentatif, seule apte à concrétiser la meilleure des sociétés possibles. Nous nous trouvons ainsi devant une démarche qui n'hésite pas à recourir à « la langue abstraite » pour analyser les formes les plus réelles de la vie ${ }^{10}$.

7 En l'an III, Sieyès se propose donc de partir de la nature humaine, de ses besoins, des moyens qui sont nécessaires aux hommes, saisis dans leur rapport pour construire le vrai ordre politico-social. Il en déduit l'importance des droits et des devoirs réciproques :

«Il y a bien longtemps que, fatigué des vains efforts des théologiens et des métaphysiciens scolastiques, dégoûté des trente premières pages de tout ouvrage philosophique sans exception, j'ai senti qu'il fallait partir d'un fait certain et non d'une supposition. L'homme naît avec des Besoins et des Moyens, de là découlent des droits et devoirs. J'ai donné en 1789 cette nouvelle base à la Déclaration des droits que le comité de constitution de l'Assemblée Nationale m'avait demandée. Aujourd'hui, il me paraît inutile de remonter si haut; un fait plus rapproché se trouve convenu et avoué de tout le monde ; c'est que tout homme doit être libre. $»^{11}$

8 Sieyès s'attache alors à la définition de la liberté humaine en fonction de ses besoins et de ses moyens. Rappelons aussi que la liberté de l'homme, selon lui, est la liberté de sa 
volonté : «La liberté ou volonté non dominée », c'est le titre de l'une des ses notes manuscrites ${ }^{12}$. Mais cette liberté doit "être corrélée avec celle d'autrui ${ }^{13}$. Ici Sieyès reprend des idées développées en 1793 dans le Journal d'Instruction sociale où il distinguait deux sortes de liberté humaine : d'abord la liberté d'indépendance, liberté originaire, lorsque l'homme "n'est point empêché par d'autres hommes de faire sa volonté ", et ensuite la liberté de pouvoir " pour exercer ou employer son pouvoir pour l'accroître de plus en plus ». Ainsi, d'une étape à l'autre, le développement de la liberté mène au perfectionnement de l'état social :

«La liberté toute seule mène-t-elle à l'état social? Prenez-la dans ses développements de pouvoir et même d'indépendance; et si vous ne voulez trahir ses intérêts, convenez que la réponse est : Oui. ${ }^{14}$

En résumé, la liberté individuelle est, selon Sieyès, directement liée à la satisfaction par l'individu lui-même de ses besoins dans des rapports de droit et de réciprocité avec ses semblables. Sieyès n'écrit-il pas :

"C'est donc par la seule voie des engagements réciproques et libres que les hommes, si nécessaires au bonheur, à la liberté les uns des autres peuvent traiter ensemble. Or, parmi les engagements féconds en fruits utiles, le plus important, le plus précieux de tous est celui qui lie la volonté commune de l'association, c'est-à-dire envers la loi." 15

De cette voie surgit la liberté étendue ou extensive qui consiste à étendre sa liberté à un maximum de choses dont l'homme veut avoir l'usage pour en jouir. Nous arrivons alors au stade ultime de la liberté représentative, propice à l'unité organisée du système représentatif. L'art social atteint son apogée sous la forme d'un «art de l'économie sociale », ou «art de la représentation ». Il s'agit bien tout à la fois d'augmenter la généralité des fins et de diminuer la dépendance des moyens, donc de produire les plus grands effets avec le moins de frais possible. L'art social devient "l'art d'usage des Droits de l'homme ou leur exercice dans une multitude d'individus $»^{16}$. C'est pourquoi, dans « l'état social représentatif », domine la figure de législateur, seul apte à traduire dans la loi les besoins du peuple, exprimés d'abord sous forme de demandes, donc seul capable de trouver les moyens de les satisfaire.

11 Ailleurs, Sieyès précise cette question essentielle des besoins humains de la façon suivante: "Dans la nature, tout ce qui a des besoins (et tout ce qui est amené à des besoins) a le droit d'y satisfaire et par conséquent des droits aux moyens essentiels pour cela $»^{17}$. D'une part, une fois admis que l'activité individuelle est limitée par l'activité des autres, il importe de préciser que la ligne essentielle séparant l'homme des autres animaux n'est autre que l'existence dans chaque individu d'un principe interne, cette volonté avec laquelle l'on s'entend et l'on s'engage. D'autre part, les Droits de l'homme proviennent de la même source, des besoins. À ce titre, ils peuvent se réduire à un seul : ne point forcer autrui, le laisser libre, déterminer sa volonté par l'offre de quelque chose qu'il préfère à ce que vous lui demandez ${ }^{18}$.

12 Le développement de l'ordre social est donc bien déterminé, selon Sieyès, par le développement de la sociabilité interindividuelle au sein même du processus de satisfaction des besoins réciproques. Sieyès écrit encore à ce sujet: «La société est fondée sur des besoins réciproques [...] Le nombre des besoins réciproques et leur intensité vous donnent la quantité de sociabilité qui existe entre les êtres $»^{19}$. La résultante du développement des besoins réciproques n'est autre que l'ensemble des engagements réciproques comprenant les rapports de droit: "Vous n'avez, vous n'exercerez d'autres droits sur les autres que d'après les engagements à votre égard $»^{20}$. 
Sieyès voit ainsi dans la progression de tels rapports réciproques les bases réelles de toute société. Et il ajoute: "Ces échanges d'engagement réciproques se trouvent partout où il y a société. $»^{21}$

Qui plus est, le seul régulateur des relations sociales est, selon lui, le mécanisme qui doit établir les rapports parmi les besoins des membres de la société, le mécanisme du marché : "Cet homme avec qui vous traitez, il vient comme vous, il cherche comme vous à profiter des avantages sociaux. Vous lui faites tout en reculant sur le marché tout ce qu'il y tient. Les avantages sociaux sont à ce prix ${ }^{22}$. En dernière analyse, ce mécanisme peut alors s'organiser dans des rapports optimaux entre les besoins de l'homme et les moyens de les satisfaire. En outre, le marché est la garantie de la liberté dans la société et la protège des ébranlement sociaux. Idée essentielle que Sieyès formule de la façon suivante : "Représentation des besoins et des moyens garantit la liberté et l'impossibilité des révolutions. Je ne vois plus de liberté dans celui à qui vous donnez $\mathrm{B}$, quand il a besoin de $\mathrm{A} »^{23}$

Il en est ainsi de la liberté et des Droits de l'homme au sein de l'état social :

"Ainsi, puisque, dans l'état social, l'homme croît en moyens moraux et physiques, et qu'il se soustrait en même temps aux inquiétudes multipliées qui en accompagnaient l'usage, il est vrai de dire que la liberté est plus pleine et plus entière dans l'ordre social, qu'elle n'a jamais pu l'être dans l'état qu'on appelle de nature. $»^{24}$

Cependant le droit naturel n'est pas propre aux besoins humains réels. Sieyès qualifie ainsi les droits naturels de « matières brutes »:

«Il faut répéter que les droits naturels antérieurs à la société ne sont que les matières brutes qui seront fabriquées et plus appropriées aux besoins individuels dans l'état de la société. Les droits naturels ont leur complément en devenant droits sociaux. Aussi faut-il présenter les Droits de l'homme en société. $»^{25}$

16 Les droits naturels sont donc imparfaits par rapport aux droits sociaux jugés plus parfaits. En effet, le droit naturel n'établit pas les garanties des engagements réciproques issus des besoins réciproques:

« Il ne s'agit plus des droits naturels dévorant [...] ses forces [...]. Il y avait des droits épurés, passés au creuset de la liberté : ils sont conséquents aux devoirs; vous n'avez en société un droit sur quelqu'un qu'autant qu'il a une dette envers vous. C'est là le droit parfait, convenu ou reconnu, et garanti socialement. Les droits imparfaits tiennent à l'état naturel, ce sont ceux auxquels il n'y a pas de garantie sur engagement : droits de bienfaisance, de secours, de politesse [...] toute garantie n'y manque pas, elle est dans les mœurs publiques et fait partie de la morale, elle est dans le pouvoir public, elle est en particulier dans les mœurs internes naturels ou acquis. ${ }^{26}$

Reste que Sieyès ne croyait pas la garantie sociale suffisante en elle-même. Il convient de la compléter par la garantie politique. En effet « la garantie politique est la caution de toutes les garanties sociales ${ }^{27}$. Il en résulte qu'il n'analyse pas vraiment l'homme naturel : il concentre plutôt son attention sur l'homme social et moral. Nous retrouvons alors les traits caractéristiques de l'individu libéral tel que Claude Gautier les a reconstitués sur la base des enseignements de l'école anglo-écossaise (Mandeville, Smith, Ferguson essentiellement): "Un individu doué de facultés actives qui le conduisent nécessairement à entrer en mouvement. Un principe de mouvement qui est tout autant conflit, activité dont une des formes sera le travail. Un individu actif pour qui les conduites, les pratiques sont autant de modalités vraies d'une composante naturelle, le principe anthropologique de l'ouverture sur autrui, sur l'extérieur. ${ }^{28}$ 
D'ailleurs Sieyès n'a-t-il pas écrit :

«L'homme a besoin d'être libre non pour être libre, mais pour exercer ou employer son pouvoir. Il a besoin d'être libre pour augmenter ce pouvoir de plus en plus [...]. Vous voyez que l'objet de la liberté, qui est l'accroissement de pouvoir ou de moyens libres, a besoin de l'état social et des toutes les institutions qui les composent. $»^{29}$

19 En résumé nous pouvons affirmer, avec Jacques Guilhaumou, que «ce principe anthropologique de la réciprocité humaine est tout aussi important pour Sieyès. Il fonde ce qu'il appelle les «besoins réciproques » et explique l'extension/généralisation du droit naturel en société, sans rupture contractualiste, par le développement des rapports réciproques de sociabilité. Ainsi se précise le fondement de sa conception du droit : ce développement est contenu jusqu'à l'accomplissement du politique dans le déploiement même de moyens aptes à concrétiser les droits de l'individu dans la nation. $»^{30}$

La croissance et la suffisance des besoins publics posent inévitablement la question du rôle de la propriété dans la formation de l'ordre social. Dans son manuscrit sur les «Bases de l'ordre social », Sieyès a fait ressortir la place centrale de la propriété au sein des Droits de l'homme :

«Si l'homme est libre, tous le sont, c'est-à-dire tous sont propriétaires inviolables de personnes et de leurs choses. Ils doivent user de l'une et de l'autre, sans empêchement de la part d'autrui. Tous les droits sont renfermés dans cette propriété personnelle et réelle. Dans cet état de liberté, les hommes ne peuvent traiter ensemble que volontairement et par voie d'échange. Ils se reconnaissent leurs droits respectifs, et par cette reconnaissance réciproque, ces droits d'abord purement naturels prennent le caractère de droits positifs. L'engagement volontaire est le principe de toute obligation positive. À côté du droit naît le devoir. Le jeu corrélatif des droits et des devoirs est l'âme de l'ordre social. " $^{31}$

21 La propriété soumet donc à elle-même tous les droits, voire les définit. Il en résulte une coexistence, dans la pensée de Sieyès, entre le principe jusnaturaliste de la réciprocité du droit, de l'extension mutuelle du droit, et le principe positif de la reconnaissance réciproque des droits de propriété, des relations de propriété. À vrai dire, Sieyès n'est pas de stricte obédience jusnaturaliste. Mais l'importance qu'il accorde au droit subjectif, sur la base d'une métaphysique du moi, ainsi que l'accent sur une réalité constituante étendue jusqu'au politique nous interdisent de le compter parmi les partisans du droit positif stricto sensu. Sa logique de pensée, en matière des droits, relève de la présence d'un droit positif de propriété au sein d'un processus d'affirmation de la valeur constituante des droits subjectifs.

Par ailleurs, Sieyès a abordé la définition libérale des Droits de l'homme par le biais d'une réfutation partielle de l'option démocratique. Il s'inscrit dans l'actualité de Thermidor où l'identification entre démocratie et jacobinisme est monnaie courante. Il oppose ainsi une tradition libérale se réclamant de Locke, Montesquieu, Voltaire, des Physiocrates, voire des Feuillants et des Girondins ${ }^{32}$, et une tradition démocratique remontant jusqu'à Rousseau et Mably dont le courant «jacobin-démocrate » se veut l'héritier ${ }^{33}$. De fait, pour Sieyès, les Droits de l'homme sont avant tout les droits de l'individu ${ }^{34}$, et non ceux d'une société prise comme une entité pouvant avoir des droits différents des intérêts individuels ${ }^{35}$.

Résumant sa position en la matière, Sieyès écrit : 
«Les Droits de l'homme sont donc antérieurs à tout. Ils ne se perdent pas en entrant en société ; au contraire, le grand objet de l'association politique est de leur donner une plus forte garantie en les mettant sous la protection de la communauté. Mais il importe de ne pas oublier que protéger les droits n'est point les créer, les accorder. Lorsqu'une autorité tutélaire sera établie dans la société, ce n'est pas d'elle que les droits découleront; ils n'auront pas leurs sources dans la loi. Au contraire, la loi, et la législation, et toute autorité publique, ont leurs sources et la raison de leur existence dans les droits ; ils étaient avant tout et tout est fait pour les servir. ${ }^{36}$ libérale de l'ordre social. C'est pourquoi Sieyès s'attarde sur les rapports entre liberté et égalité, étant admis "que la liberté gagne de plus en plus dans l'état social, et que toutes ces lumières, tous les progrès sont au profit de la liberté. Mais cette vérité serait anéantie si tous n'étaient pas égaux en droits $»^{37}$. Alors il souligne d'une part que "les besoins réciproques ne sont cause de sociabilité qu'entre égaux $»^{38}$. D'autre part, l'égalité ne doit pas restreindre la liberté, ajoute-t-il: «L'égalité qu'il nous faut ne blesse point la liberté $»^{39}$. De fait, repensant l'expérience historique du gouvernement révolutionnaire et identifiant ce phénomène politique à la démocratie directe ou pure, Sieyès dénonce la réduction de la liberté propre à l'égalité en démocratie: « $\mathrm{La}$ démocratie [...] ne donnera que le minimum de liberté individuelle. Car c'est le sacrifice complet de l'individu à la chose publique $»^{40}$. L'égalité démocratique mène non seulement à l'égalité sociale, mais aussi à la nationalisation de la propriété : «Combien de gens confondent l'égalité démocratique avec l'unité de propriété ? »11 Certes, «les propriétés publiques sont de l'égalité ${ }^{42}$, mais en fin de compte, les propriétés publiques ne peuvent pas exister sans propriété privée : "Si vous dégouttez du travail particulier en lui arrachant un trop fort parti de produit, vous diminuez le travail et [...] des propriétés publiques dépérissent avec celles des particuliers. $»^{43}$

Cependant si l'égalitarisme mène au dépérissement de l'ordre social, a contrario l'inégalité sociale est une condition nécessaire de la prospérité : «L'esprit public tend au niveau de l'égalité tandis que l'industrie [...] visant à l'inégalité enrichit la société et tourne à l'avantage de tous. $»^{44}$

26 Comment Sieyès définit-il ensuite une égalité qui ne restreint ni la liberté, ni la propriété ? À l'exemple de la Révolution de 1789, il identifie tout d'abord l'égalité à la suppression des privilèges au nom de l'autonomie de l'individu et de son insertion dans la division du travail. La définition de l'égalité est alors la suivante :

«Mais quelle que soit la force commune, elle doit être impartiale, elle doit s'offrir également à tous, c'est un soutien commun où chacun puise suivant ses forces ou ses besoins et qui n'en privilégie aucun en particulier. Voilà l'égalité. ${ }^{45}$

27 Il est question ici d'une égalité en droit, non contradictoire avec l'inégalité sociale, voire qui la suppose, au titre de l'impartialité de la loi issue de la souveraineté nationale :

«C'est l'égalité ou l'impartialité elle-même de la loi qui fait prospérer les différentes inégalités [...] des membres de la société. Car en m'assurant ma propriété, il est clair qu'elle ne permet pas de l'augmenter et de la rendre par conséquent relativement plus inégale. Il faudrait que la loi fut inégale ou partiale pour ne pas favoriser les différences ou inégalités individuelles. $»^{46}$

C'est ainsi que "l'égalité n'est que la non-inégalité du fait de la loi ${ }^{47}$, une égalité négative au sens où «l'égalité civile est donc une juste correction de l'inégalité des moyens $»^{48}$. Dans le même temps, Sieyès se prononce contre la société égalitaire 
démocratique fondée sur la communauté des citoyens : «C'est une loi commune et non une vie, une propriété, une existence commune que suppose l'union sociale $»^{49}$. Sieyès prend alors soin de distinguer deux séries lexicales distinctes: les mots égalité, égaux, équifier s'opposent ainsi à égaliser, égalisation, égaliseur mots qui désignent le projet démocratique égalitaire.

Ainsi Sieyès, fidèle à la tradition libérale qui sépare strictement droits politiques et droits civils de l'individu, s'oppose à une tradition démocratique qui met l'accent sur l'unité des droits politiques et civils : le citoyen, d'après cette dernière tradition, est celui qui possède la totalité des droits politiques et civils. Et Sieyès d'ajouter :

«Le droit dont chacun jouit de recourir à la garantie de la loi et de l'autorité tutélaire est proprement le droit civil. Si l'on veut parler avec précision, ce droit médiat n'ajoute rien aux droits primitifs, il en est une conséquence naturelle [...] c'est plutôt un service de garantie et de régularisation dans l'exercice des droits naturels. $\|^{50}$

Qui plus est, les droits politiques sont aussi médiats, ils garantissent en effet aux individus la sûreté par rapport aux pouvoirs publics :

«Mais où est-elle cette garantie contre les pouvoirs établis pour protéger, s'ils s'avisent de trouver leur force relativement irrésistible contre les droits individuels? Elle est dans l'existence et l'activité des droits politiques. Puisque l'homme est libre, qu'il ne s'oblige que par voie d'engagement volontaire, qu'il ait le droit de prononcer sa volonté politique, comme sa volonté civile. $»^{51}$

Il s'agit bien de montrer comment la liberté individuelle se détermine pleinement dans «l'ordre politique ». Par ailleurs, Sieyès soutient que l'égalité politique mise en œuvre par les « classes disponibles » pour les affaires du gouvernement apporte une garantie d'égalité à tous. La politique est donc un métier réservé à une élite garante de l'égalité positive pour tous, alors que l'égalité civile est une égalité seulement négative du fait de la positivité de l'inégalité des moyens.

Nous pouvons donc voir en quoi la position de Sieyès en matière de droits de l'individu se différencie de celle des autres membres de la Convention thermidorienne. Ces derniers ont voulu unifier, dans la Constitution de l'an III, les droits politiques et les droits civils ; à ce titre, ils ont opéré une confusion entre la citoyenneté et la fonction électorale. En effet, l'article 8 de la Constitution subordonne au paiement d'une contribution directe non seulement l'entrée dans les assemblées primaires, mais aussi la possession de la qualité de citoyen. Ainsi une partie des Français a été privée des droits politiques et civils.

En revanche, Sieyès, toujours fidèle à la Constitution de 1791, revêt tous les Français des droits civils, donc de la qualité de citoyen. En matière de droits politiques, il se prononce pour l'établissement de listes d'éligibles " comme en 1789 », mais il ne stipule pas l'existence d'un cens pour les électeurs et les éligibles. La position de Sieyès s'avère ainsi plus démocratique que celle de la majorité thermidorienne. Si le système représentatif, voulu par Sieyès et la Convention, admet le suffrage restreint, rien, dans sa position, à la différence de la majorité de ses collègues à la Convention, ne s'oppose, dans le principe, à ce que le suffrage universel s'applique dès que l'on considère que tous les citoyens (femmes comprises) sont aptes, par leur capacité et leur éducation, à voter et à être éligibles.

Sieyès prête une grande importance à l'organisation du pouvoir politique. L'explication des origines et de la formation du pouvoir spécifique d'un régime politique est l'un des 
meilleurs moyens de susciter l'adhésion des citoyens à la politique gouvernementale, donc de fonder la légitimité d'un régime politique. En période révolutionnaire, c'est-àdire de crise permanente de la légitimité, les interrogations sur la légitimité d'un régime politique sont d'une grande importance.

Pour Sieyès, les origines du pouvoir résident dans le fait de l'association politique, ellemême fondée sur le déploiement de la volonté générale :

«L'association politique est bien l'ouvrage des associés. La loi, qui doit les obliger, ne peut être que l'expression de la volonté générale. Je dis plus, la création de l'établissement public et de la Constitution qui l'organise, ne peuvent être que le produit de cette même volonté. $~^{52}$

Considérant la fameuse thèse de Rousseau selon laquelle la loi est l'expression de la volonté générale, Sieyès définit ainsi la démocratie pure : «Les associés se réuniront, s'il sont en petit nombre. Ils discuteront leurs besoins politiques, et conviendront des moyens les plus propres à y pourvoir. C'est la Démocratie dans son origine $»^{53}$. Il en déduit, fort classiquement, qu'une telle démocratie n'est possible que sur de petits territoires. Pour les grands États, deux possibilités demeurent, soit les réunions locales, soit la nomination de députés à une Assemblée centrale ${ }^{54}$.

C'est alors que le système représentatif, tel que le conçoit Sieyès, pénètre l'ordre social et l'organise ; il a pour fondement la division des travaux. Sieyès décrit les origines du système représentatif dans les termes suivants :

«La division des travaux, des professions, etc., n'est que le système représentatif qui s'établit de lui-même; il marche avec les progrès de la société qu'il anime. Il est le plus favorable à la production des richesses, à la commodité des échanges et au mouvement général des affaires. Il s'est presque emparé de toutes les actions des hommes. ${ }^{55}$

38 À l'idéal démocratique de Rousseau, Sieyès oppose donc les vertus du système représentatif, ce véritable concert social :

«Une mystérieuse phrase de Rousseau s'oppose seule à ce concert, la volonté générale ne peut point être représentée. Pourquoi pas? Il ne s'agit pas ici de la volonté entière de l'homme et les exemples sont nombreux de particuliers et de puissances qui traitent sur tel ou tel point, par voie de procuration. D'ailleurs, dans l'état social dont il s'agit, celui qui refuse à se soumettre à l'engagement pris par son représentant est libre de quitter l'association. $»^{56}$

Le système représentatif est donc le seul qui puisse assurer la liberté pleine et entière («Loin de compromettre la liberté, le système représentatif lui est favorable ») (57 $^{57}$ opposition à la démocratie pure. De fait, la démocratie "brute " présente quatre défauts : les passions y sont trop présentes; les votes n'y sont point contenus dans une sphère étroite, par absence d'une procuration limitée; la confusion règne entre pouvoir constituant et pouvoir constitué ; la majorité moins éclairée, disposant de tous les pouvoirs à la fois, peut en abuser et vicier le système politique en mettant la minorité sous le joug. Reste aussi que la nécessité «démocratique » de maintenir de petites unités de population, de richesses insuffisantes, met le régime démocratique à la merci de ses voisins.

Cependant Sieyès admet une certaine démocratisation du système représentatif, dans la mesure où le seul mode acceptable d'expression de la volonté générale fait partie intégrante de ce système : 
« Il arrivera donc que les assemblées primaires se borneront à nommer des députés dont la réunion formera une assemblée nationale législative propre assurément à prononcer cette volonté générale que nous cherchons " $^{58}$

41 Entre la Convention thermidorienne, qui veut protéger le régime représentatif des dangers de la démocratie pure, et Sieyès, partisan d'une élection directe des députés, et proposant de doter le système représentatif d'éléments issus de la démocratie politique, la différence est sensible. De fait, Sieyès recherche, à la différence de ses collègues députés, les moyens de surmonter la méfiance envers le système représentatif issu de la Constitution de 1791. Nous savons que la Constitution de 1791 a favorisé la prédominance de "l'absolutisme parlementaire", sorte de souveraineté parlementaire source d'une large méfiance des couches populaires de la société à l'égard des représentants de la Nation ${ }^{59}$. Sieyès veut donc éviter un double écueil : d'une part la faiblesse du système représentatif à base censitaire issu de la Constitution de 1791 ; d'autre part les surenchères « démagogiques » du régime politique prévu par la Constitution de 1793.

Il maintient donc l'idée jacobine initiale d'« unité d'action» associée à «la centralité législative », mais il critique l'instauration de «l'action unique» par les Montagnards au nom du rapprochement entre le peuple et ses représentants ${ }^{60}$. De même, il lui importe que la procuration des députés ne soit pas impérative, " en ce sens qu'ils ne seraient que de simples porteurs d'un vote fixe, déterminé d'avance. Puisqu'il s'agit de parvenir à former une volonté générale, il faut leur confier le pouvoir de traiter, de se rapprocher, enfin de convenir ensemble. $»^{61}$

Il met également en garde contre toute extension excessive de la souveraineté et de la soumission de la société civile au pouvoir politique :

«Cette procuration n'est pas illimitée; outre que rien n'est illimité dans la nature, il est clair que des procureurs fondés ne peuvent point s'occuper d'affaires hors de leur mission spéciale. On ne doit pas croire que les associés politiques aient voulu mettre en commun toutes leurs affaires, leurs personnes et leurs choses [...]. Ils ne mettent en commun qu'une action ou une valeur limitée par l'importance des besoins communs et réputés suffisant pour faire fleurir l'association. On n'a point voulu créer une Ré-totale, mais seulement une République. ${ }^{62}$

Une seconde fois donc $^{63}$, Sieyès s'en prend à la "Ré-totale ", c'est-à-dire à une extension excessive de l'égalité, et donc de la souveraineté. Il se pose la question: "Mais qu'est ce que la souveraineté ? Les associés mettent-ils en commun et font-ils représenter la totalité de leurs moyens?» Et il répond: «Non assurément. Ainsi la souveraineté prise pour un pouvoir suprême qui dominerait et embrasserait tout n'existe pas [...] si la Constitution sépare les pouvoirs, si chacun d'eux borné à sa mission spéciale ne peut en sortir sans usurpation et sans crime, où placer l'idée gigantesque de souveraineté ? " ${ }^{64}$ Donc « la souveraineté est enfermée dans les limites d'un pouvoir public ${ }^{65}$, les droits du souverain sont légitimes à l'intérieur du cadre de système représentatif. L'extension de la souveraineté, propre à la démocratie pure, revient à exercer la tyrannie de la majorité sur la minorité. Une seule issue en fin de compte au risque d'une tel glissement démocratique excessif, l'adoption d'une Constitution $^{66}$ :

«Or, dès que nous supposons la volonté sociale divisée en majorité et minorité, si la majorité qui fait la loi n'a pas de freins, elle peut devenir tyrannique, ou tout du moins despotique envers la minorité. Ce ne peut pas être là le but de l'association ; il est donc démontré que pour tout esprit droit, pour tout /individu/ digne d'être libre qu'il doit exister avant toute loi faite par la simple majorité une volonté 
unanime qui donne un frein aux législations à simple majorité et lui rend impossible la tyrannie et même le despotisme. Je dis que cette volonté unanime et primitive fait parti essentiel ( ?) de l'acte d'association ou la précède [...]. Je dis que la division des pouvoirs et leur organisation, c'est-à-dire la constitution (car ce n'est pas autre chose) est une loi fondamentale antérieure à la loi faite à la simple majorité. Obéir à la constitution fait partie de l'engagement primordial de chaque associé individuellement." 67

Sieyès qualifie $d^{\prime}$ 'sétablissement public» la sphère politique issue du système représentatif, donc instituée par la volonté nationale: «Composée de choses et de personnes, etc., cette grande institution qui doit sa création à la Volonté Nationale en reçoit aussi de son organisation $»^{68}$. La formation de "l'établissement public» correspond à la nécessité de satisfaire les besoins publics : « On ne saurait pourvoir aux besoins communs de la société sans un établissement public $»^{69}$

À vrai dire, Sieyès ne rejette pas complètement l'idée de démocratie. Il note à ce sujet : «il faut, sans doute, de la démocratie dans un bon système social $»^{70}$. Dans un autre manuscrit, notons aussi le résumé des idées de Sieyès en la matière par Hippolyte Fortoul: "On ne saurait pourvoir aux besoins communs de la société sans un établissement public $»^{71}$

La véritable démocratie est donc la démocratie sociétale ou civile: "C'est là que l'homme peut valoir et que l'État recueille tout ce qu'il peut recueillir $»^{72}$. Certes, « l'établissement public n'est pas la démocratie, c'est un corps élevé par la démocratie, sur la démocratie pour les besoins publics». Aussi, «on a tort de confondre avec la démocratie qui est l'existence sociale et l'action commune cette machine politique qui recueille, digère et renverse sur tous les moyens des besoins communs. La démocratie est dans les citoyens et dans leur masse ; dans l'établissement public, au contraire, il ne peut y avoir que unité et organisation ».

Il faut donc envisager, pour Sieyès, une démocratisation préalable de l'ordre social, garante de l'égalité des droits civils :

«Ce n'est que dans la démocratie que l'établissement public est sur sa base, sur une véritable base [...]. À présent, dites-moi quelle est l'idée de ceux qui veulent démocratiser chaque action politique dans le corps de l'établissement public? Autant vaut démocratiser chaque action civile ou individuelle dans la masse des citoyens. Démocratiser la justice, la police, la guerre, les finances, etc., c'est démocratiser l'art de /.../ la construction. $»^{73}$

Cependant le point crucial de "l'établissement public» demeure, pour Sieyès, l'organisation des pouvoirs. Il n'a jamais été partisan de la théorie de la division et de l'équilibre des pouvoirs, dans la mesure où «le despotisme cède la place non pas à l'équilibre des pouvoirs, mais au concours des pouvoirs $»^{74}$. Il critique ainsi les jacobins d'avoir insuffisamment tenu compte de la dialectique unité-multiplicité :

«En fait de gouvernement, et plus généralement en fait de constitution politique, unité toute seule est despostisme, division toute seule est anarchie; division avec unité donne la garantie sociale sans laquelle toute liberté n'est que précaire. ${ }^{75}$

Soucieux donc d'établir cette "unité organisée », Sieyès distingue, comme en 1789, le pouvoir constituant du pouvoir constitué ${ }^{76}$. La Nation peut, en vertu du pouvoir constituant de sa délégation de pouvoir à des représentants, se doter d'une Constitution posant les règles de fonctionnement de la représentation législative et des pouvoirs constitués. 
51 D'une part, l'exercice de ces deux pouvoirs doit être clairement distinct, sinon un pouvoir exécutif peut de lui-même en contredire un autre :

«Les pouvoirs publics doivent être divisés, limités, organisés, en un mot constitués pour remplir leur destination. Si un Corps pouvait se constituer lui-même ou toucher à sa constitution, bientôt il changerait de nature, il se rendrait propre à tout envahir, et à dévorer ses créateurs. $\gg^{77}$

D’autre part, le pouvoir constituant est inaliénable, mais délégable dans une situation précise :

«Une nation qui délègue son pouvoir constituant s'expose à perdre sa liberté et à changer d'état presque à son insu. Elle doit s'en réserver l'exercice dans ses assemblées primaires, mais elle peut confier à des délégués spéciaux le soin de lui présenter les projets d'amélioration que l'expérience et le temps ont rendu nécessaires. ${ }^{78}$

Ainsi les assemblées primaires se trouvent à la base de la pyramide politique :

«Les assemblées primaires sont le véritable fondement de l'état public; elles en contiennent tous les éléments; elles se composent de tous les citoyens qui ont la volonté et capacité d'y assister. $»^{79}$

Sieyès situe au sommet de cette pyramide "les grands pouvoirs constitués » dont il conçoit le principe central dans la séparation de leurs fonctions :

«Il est aujourd'hui généralement reconnu que les grands pouvoirs constitués doivent aussi être séparés ; mais on ne sent pas assez le besoin de rendre l'exercice de leurs différentes fonctions incompatibles dans la même personne. Ce mélange entraîne une partie des inconvénients que l'on a voulu éviter par la division. ${ }^{80}$

Sieyès commence alors par distinguer, au niveau du pouvoir suprême, le pouvoir législatif. À ce titre, héritier du légicentrisme de 1789 comme tant d'autres, il n'est pas si étranger qu'on le croit au projet unitaire des Montagnards, formulé dans l'expression de « centralité législative ». Il le définit cependant à sa façon :

«Le pouvoir législatif n'est pas confié à la seule chambre des députés. On a senti le besoin de le diviser lui-même en trois parties: le chef de l'État, le sénat et la chambre des députés; aucune de ces trois chambres ne peut se dire séparément corps législatif, aucune se croire législateur ou représentant législatif. Ces qualités sont indivises et n'appartiennent qu'à la réunion des trois. Ce n'est pas que tous ne soient représentants pour ce qu'ils ont à faire. On représente toujours ceux pour qui on agit. $»^{81}$

Dans l'établissement public, il ne peut y avoir que des représentants. Il revient donc sur sa position énoncée dans sa polémique contre Barnave et d'André en 1790, qui insistait alors sur la possibilité de donner au roi la qualité de représentant héréditaire du peuple, à la différence des fonctionnaires publics. Le fait que l'Assemblée constituante et la Constitution de 1791 puissent reprendre cette position, en instaurant une inégale légitimité entre un pouvoir législatif électif et un pouvoir exécutif héréditaire, marque, pour Sieyès, le défaut principal du régime antérieur de «monarchie constitutionnelle ». Sieyès considère, dans un texte de 1791, que tous les représentants du peuple sont ses mandataires, et qu'à ce titre, le roi ne peut être un représentant :

«Vous dites, Messieurs, [...] que si l'on peut attaquer la qualité de représentant du peuple donné au roi par les comités, on peut aussi sous quelque rapport la défendre. Il me semble en cela que [...] personne ne conteste que tous les pouvoirs, toutes les fonctions publiques venant du peuple ne soient exercés par des mandataires qui à ce titre peuvent se dire représentants. $»^{82}$ 
Ainsi, à l'exception du roi, «tout homme qui exerce un pouvoir public même dans l'ordre exécutif est véritablement à ce titre représentant du peuple ${ }^{83}$. Une fois exclu le roi de la représentation, le chef de l'État, ou «Grand électeur » est une réalisation pratique de la figure du législateur chargé de sauvegarder la Constitution. À cette fin, le «Grand électeur » incarne sa fonction au nom d'un double principe, l'éligibilité de son poste (qui manque au roi) et la succession ou l'hérédité. Certes Sieyès n'a pas explicité en l'an III cette idée de "Grand électeur » auprès de ses collègues, il n'en parlera que plus tard. Mais il pose déjà le problème à résoudre dans une note manuscrite: «Comment se procurer dans un État tous les avantages de l'élection d'un chef, sans avoir à en redouter les inconvénients, et sous les avantages de l'hérédité sans aucun de ses innombrables dangers $»^{84}$

est dans des notes plus tardives que Sieyès explicite son idée de "Grand électeur » qu'il appelle "monarque » et considère comme "le représentant honorifique de la nation ${ }^{85}$. Il témoigne aussi, dans une autre note au titre éloquent, "Élections et fonctions du monarque, grans électeur", de sa sympathie pour une "monarchie constitutionnelle » en tant que partie intégrante d'un système représentatif apte à clore le processus révolutionnaire. Mais, tout à la fois soucieux de défendre les avantages d'un gouvernement monarchique et de tenir compte de la diffusion des valeurs républicaines dans l'opinion publique, Sieyès n'hésite pas à classer la figure du monarque sous le titre de républicain ${ }^{86}$.

Sieyès critique aussi l'usage du mot « le gouvernement » dans la théorie du despotisme. Pour lui, «l'idée du gouvernement reste particulière et intérieure à l'établissement public. Quelle extension on a pourtant donné à ce mot! La manie de gouverner recèle l'ambition du despotisme. Les réflexions analogues sont applicables au mot pouvoir exécutif. Qui est-ce qui exécute les lois? Ce n'est ni le chef de l'État, ni les fonctionnaires, c'est bien le citoyen ${ }^{87}$. C'est pourquoi, face à l'abus du mot et de la chose, le mécanisme politique doit être réduit à un état minimal : « Il ne doit y avoir dans un État bien ordonné d'autres institutions que celles de l'établissement public réduit à son minimum. Laissez tout le reste à l'exercice des droits particuliers. ${ }^{88}$ Mais l'État minimal ne signifie pas, pour Sieyès, un État inactif. Au contraire, dès 1789 il attribue à la compétence du pouvoir exécutif la régulation des domaines suivants de la vie de la société : l'éducation, la justice, l'économie et l'assistance sociale ${ }^{89}$.

En fin de compte, ce sont donc la Nation et ses représentants qui créent l'espace politique, et surtout « l'établissement public ». De là, il n'est pas mauvais d'insuffler un indéniable caractère démocratique au régime représentatif: «Il est bon que la législature puisse être souvent rafraîchie d'esprit démocratique »

Dans un de ses discours à la Convention en l'an III, Sieyès décrit finalement le système représentatif dans les termes suivants :

«L'action politique dans le système représentatif se divise en deux grandes parties : l'action ascendante, l'action descendante. La première embrasse tous les actes par lesquels le peuple nomme immédiatement ou médiatement ses diverses représentations, qu'il charge séparément de concourir, soit à demander ou à faire la loi, soit à la servir dans son exécution quand elle est faite. La seconde embrasse tous les actes par lesquels ces divers représentants s'emploient à former ou à servir la loi. Le point de départ de ce mouvement politique dans un pays libre ne peut être que la nation dans ses assemblées primaires; le point d'arrivée est le peuple recueillant les bienfaits de la loi. $»^{90}$ 


\section{NOTES}

1.Je remercie Françoise Brunel et Jacques Guilhaumou pour l'aide qu'ils m'ont apportée dans l'établissement de la version française de ce texte.

2. Euvres de Sieyès, notes liminaires par Marcel Dorigny, Paris, Edhis, 1989, 3 volumes, tome3, documents 40 et 41 .

3.Sous la cote $284 \mathrm{AP}$.

4.A.N., 284 AP5 d.1 (4). Ce manuscrit a été publié par Pasquale Pasquino, en annexe de son ouvrage, Sieyès et l'invention de la Constitution en France, Paris, Odile Jacob, 1998.

5.« Bases de l'ordre social... an 3 », préface.

6.Id.

7.Sur la Déclaration des droits en général, A.N., 284 AP5, d.1 (5). Voir la publication partielle de ce manuscrit par Christine Fauré dans Les déclarations des droits de l'homme de 1789, Paris, Payot, 1988.

Annales historiques de la Révolution française, 319 | janvier-mars 2000 
8.Voir J.L. Marion, Le prisme métaphysique de Descartes, Paris, 1986.

9.A.N., 284 AP 2 D.2.

10.Voir sur ces divers points l'article synthétique de Jacques Guilhaumou, « Nation, individu et société chez Sieyès », Genèses, 26 avril 1997.

11. "Bases de l'ordre social... », op. cit.

12.A.N., 284 AP5 d.1 (3), « Idée d'une Constitution (an III) au Comité des Onze ».

13. "Sur la Déclaration des droits ", op. cit.

14.Journal d'Instruction sociale, $\mathrm{N}^{\circ}$ 11, 8 juin 1793, p. 48, Paris, reprint Edhis, 1981.

15.Id., pp. 47-48.

16. "Idées d'une Constitution (an III). Droits de l'homme », op. cit.

17. "Sur les Déclarations des droits en général. Droits de l'homme », op. cit.

18.Id.

19.Id.

20. «Sur les Déclarations des droits. Droits de l'homme A », op. cit.

21. "Sur la Déclaration des droits en général », op. cit.

22.« Idées d'une Constitution... Devoirs », op. cit.

23. "Principes d'une Constitution, l'an III », A.N., 284 AP 5, d.1 (6).

24.Journal d'Instruction sociale, op. cit., p. 42.

25. "Idées d'une Constitution (an III). Droits de l'homme ", op. cit.

26.«Sur les Déclarations des droits », op. cit.

27.Id.

28. Claude Gautier, L'invention de la société civile. Lecture anglo-écossaise (Mandeville, Smith, Ferguson), Paris, PUF, 1993, p. 145.

29. « Idées d'une Constitution... ", op. cit.

30.« Nation, individu et société chez Sieyès », op. cit., p. 13.

31. "Bases de l'ordre social... », op. cit., § 2.

32.Les Girondins, à la différence des Feuillants, ont accepté de faire de la propriété un droit social, dérivé du contrat fondateur de la société et non naturel antérieur à ce contrat ; ils étaient également partisans de la démocratisation du régime représentatif en rupture avec le libéralisme classique des Feuillants. Voir notre ouvrage (en russe), Les Feuillants. Des origines du libéralisme français, Moscou, 1999.

33. Voir Lucien Jaume, Le discours jacobin et la démocratie, Paris, Fayard, 1989, et le colloque Mably sur La politique comme science morale, Introduction de Florence Gauthier, Bari, Palomar, deux volumes, 1995-1997.

34. "Idées d'une Constitution. La fin de l'ordre politique est la liberté individuelle », op. cit.

35.« La chose publique est faite pour l'individu, et non l'individu pour la chose publique ", A.N., 284 AP5 d.1 (3). « Idées d'une Constitution. Bases et fin de la société respect de la liberté individuelle ».

36. "Bases de l'ordre social... », § 3, op. cit.

37. "Idées d'une Constitution... », op. cit.

38. «Sur la Déclaration des droits en général », op. cit.

39.« Contrepoids ", A.N., 284 AP5 d.1(6).

40.« Idées d'une Constitution. Bases et fin de la société n'est pas unité de propriété », op. cit.

41.Id.

42.Id.

43.Id. 
44. "Idées d'une Constitution. Propriété publique, égalité » op. cit.

45. « Contrepoids », op. cit.

46.Id.

47. "Idées d'une Constitution. Égalité », op. cit.

48. "Égalité », A.N., 284 AP5 d.1 (2). Pasquale Pasquino analyse l'importance du couple égalité/liberté chez Sieyès dans son ouvrage, Sieyès et l'invention de la Constitution en France, op. cit. Jacques Guilhaumou précise ce qu'il en est de l'usage de la négation au sein des contextes définitoires de l'égalité dans « Un usage négatif d'égalité/liberté/ égalité chez Sieyès ", In/égalités/s. Usages lexicaux et variations discursives, P. Fiala éd., L'Harmattan, 1999.

49.Id.

50.« Bases de l'ordre social.. »., § 3, op. cit.

51.Id.

52.« Bases de l'ordre social... », § 6, op. cit.

53.Id., § 7.

54. "Deux moyens se présentent, l'un de faire des réunions partielles dans différentes localités, l'autre de nommer des députés pour une assemblée centrale », id., § 8 .

55.Id.

56.Id., § 8 .

57.Id., § 9.

58.Id., $\S 10$.

59.Il est ici possible de parler d' " absolutisme parlementaire » à condition de préciser que les députés demeurent dans le cadre de la délibération constitutionnelle. Voir sur ce point François Furet et Ran Halévi, La monarchie républicaine. La Constitution de 1791, Paris, Fayard, 1996.

60.Sur le projet montagnard, voir Françoise Brunel, 1794. Thermidor. La chute de Robespierre, Bruxelles, Complexe, 1989.

61. «Bases de l'ordre social... », op. cit.

62.Id.

63.Une première fois dans « Contre la Ré-totale en 1792 », A.N., 284 AP5 d.1 (1).

64.« Bases de l'ordre social. Conclusion », op. cit.

65.« Principes d'une Constitution. L'an 3. Limites de la souveraineté », A.N., 284 AP5 d1(6).

66. “C'est à la Constitution à nous garantir notre liberté ", A.N., 284 AP 5 d.1 (6).

67.A.N., 284 AP 5 d.1(6).

68. « Bases de l'ordre social... », § 12, op. cit.

69.Id.

70.Id., § 9 .

71. «Sur la Déclaration des droits en général. » Fortoul est le premier chercheur, au XIXe siècle, qui a consulté les manuscrits personnels de Sieyès.

72.Dans la note séparée « démocratie », A.N., 284 AP5 d.2, et pour les citations suivantes.

73.Id.

74. "Sur la Déclaration des droits en général », op. cit.

75.Opinion de Sieyès sur plusieurs articles..., Euvres, volume 3, op. cit., p. 3.

76.« Ainsi la division des pouvoirs la plus nécessaire à établir est celle du pouvoir constituant et des pouvoirs constitués », « Bases de l'ordre social », §12, op. cit. 77.Id. 
78.Id.

79.Id., § 14 .

80.Id., $\S 13$.

81.Id., § 15 .

82.A.N., 284 AP 4 d. 5(1).

83.A.N., 284 AP 4 d.5 (2).

84. «Bases de l'ordre social... », § 7 (6), op. cit.

85. "Couronne », A.N., 284 AP5 d.3 (3).

86. "Élection et fonction du monarque, grand électeur » A.N., 284 AP5 d.2 (5).

87.« Bases de l'ordre social... », § 16.

88.Id.

89.« Idée exacte et précise des pouvoirs publics dont l'ensemble forme la Royauté en

France », § 1 « Du pouvoir exécutif », A.N., 284 AP4 d.5.

90.Opinion de Sieyès sur plusieurs articles..., Euvres, op. cit., volume 3, p. 3.

\section{RÉSUMÉS}

La présente étude est consacrée à l'analyse des idées politiques de Sieyès en l'anIII, au moment où sa théorie constitutionnelle se cristallise. Elle met tout particulièrement en valeur les liens entre les idées sociopolitiques de Sieyès et les traditions philosophiques des Lumières. En fin de compte, l'auteur insiste sur un aspect souvent méconnu de la pensée de Sieyès, sa prise de parti pour le démocratisme au sein du système représentatif dont il propose une vision achevée en l'an III.

\section{Political Order according to Sieyès in Year III.}

The present study is devoted to the analysis of Sieyès's political ideas in Year III, when his constitutional theory was taking shape. It highlights the links between Sieyès's socio-political ideas and the philosophical traditions of the Enlightenment. Finally, the author emphasises an often neglected aspect of Sieyès's thinking, his commitment to democratism within his vision of the representative system which reached maturity in Year III.

\section{L'ordine poilitico in Sieyès nell'anno III}

Il presente studio è dedicato all'analisi delle idee politiche di Sieyès nell'anno III, nel momento in cui la sua teoria costituzionale si cristallizza. Vengono messi in particolare risalto i legami tra le idee socio-poloitiche di Sieyès e le tradizioni filosofiche dei Lumi. Infine l'autore insiste su un aspetto spesso mal conosciuto del pensiero di Sieyès, la sua adesione al democratismo in seno ad un sistema rappresentativo di cui egli propone una visione compiuta nell'anno III.

Este estudio analiza las ideas politicas de Sieyès en el año III, cuando se cristaliza su teoría constitucional. Se puede ver en particular los lazos entre sus ideas sociopolíticas y las tradiciones filosóficas de la Ilustración. Al final, el autor insiste en un punto desconocido del pensamiento de Sieyès: su adhesión al democratismo en el seno del sistema representativo del que propone una visión acabada en el año III. 
Die politische Ordnung bei Sieyès im Jahre III.

Dieser Aufsatz widmet sich der Untersuchung von Sieyès' politischen Gedanken im Jahre III, als seine konstitutionelle Theorie festliegt. Er zeigt insbesondere die Verwandtschaft zwiscuen Sieyès' sozialpolitischen ldeen und der philosophischen Erbschaft der Aufklärung.

Schließlich macht der Autor auf eine oft unbekannte Seite von Sieyès' Anschauung anfmerksam, nämlich seine Parteinahme für den Demokratismus innerhalb des parlamentarischen Systems, dessen vollendetes Gesamtkonzept er im Jahre III entwirft.

\section{AUTEUR}

ANDREİ TYRSENKO

Université de Moscou 